\title{
Implantable Prostheses for the Inner Ear
}

\author{
Christina Goodvin ${ }^{1,2}$ and Edward J. Park ${ }^{1}$ \\ ${ }^{1}$ Department of Mechanical Engineering, University of Victoria \\ PO Box 3055 STN CSC, Victoria, BC, Canada V8P 5C2 \\ ${ }^{2}$ Epic Biosonics Inc., 4464 Markham Street \\ Victoria, BC, Canada V8Z 7X8 \\ cgoodvin@epicbiosonics.com,ejpark@me.uvic.ca
}

\begin{abstract}
The aim of our research is to develop a proof-ofconcept implantable vestibular prosthesis. Such a device would be capable of 6 DOF motion sensing based on MEMS technology, to mimic vestibular functions, and provide stimulus to the brain. In this paper, the feasibility of a vestibular prosthesis is reviewed.

Epic Biosonics is in the process of developing the next generation of multi-configuration, flexible, biocompatible, micro-electrode arrays as neural stimulators for cochlear implants. Epic and the University of Victoria (UVic) are now investigating the concept of applying both Epic and MEMS technology towards building a vestibular prosthesis, i.e. vestibular equivalent a cochlear implant, to actively address balance disorders.
\end{abstract}

\section{Introduction}

The purpose of a neural prosthesis is to replace or substitute neural functions that have been impaired due to neurological disorders or trauma. Neural prostheses are emerging as a viable method in restoring impaired neural functions to patients due to the recent advances in neural engineering, This research area is being driven by a confluence of technological developments, including better understanding of the anatomy and function of nerve fibers and the availability of new micro-electrodes for interfacing to those fibers [1]. The most well-known implantable neural devices are the pacemaker and the cochlear implant. This paper focuses on the neural stimulation of the cochlea and how that technology can be applied to the second system of the inner ear, the vestibular system.

The purpose of our research is to create a viable implantable neural device for the vestibular system, the part of the inner ear that senses the body's placement and motion in space in relation to gravity. Using Micro-Electro-Mechanical-Systems (MEMS) technology and flexible biocompatible micro-electrode circuitry, we are exploring an implantable vestibular prosthesis that is able to stimulate the vestibular nerve with the electrical equivalent of healthy vestibular stimulus in such a way that the brain can correctly interpret the signals as balance information. The purpose of this paper is to provide a review on the current state of vestibular prosthetic technology and discuss some of the challenging issues involved in creating a vestibular implant.

\section{Balance Disorders}

Balance is the ability to maintain a stable upright position and make purposeful movements, by resisting the effects of gravity. The human balance system is a complex system that a combination of three main systems: vision, proprioception and vestibular, which is comprised of balance organs in the inner year. The coordination of these systems is managed by the brain. Vision allows our brain to calculate our body's relationship to the objects we see. The proprioception system - pressure signals from the joints, skin and muscles (i.e. the general sensory system) - also sends information to the brain about body (and head) posture. The vestibular system sends signals to the brain about body movement in relation to gravity and inertial acceleration.

It is the coordination of between above three systems that allows the brain to recognize the body's posture and movement relative to other objects, surfaces and gravity, which in turn signals the body to make postural adjustments to maintain balance. Whenever there is a failure in any of these systems, the result is a disruption of balance. In its most severe form, balance disorders physically interfere with a person's everyday functional abilities, as well as psychologically, due to an increase in risk of falling. For the elderly, falling due to balance disorders is a leading cause of injury and death [2]. Balance 
disorders can be due to problems in any of the following four areas [3]:

- Peripheral vestibular disorder, a problem in the vestibular organs (utricle/saccule/ampulla) in the inner ear;

- Central vestibular disorder, a problem in the brain or its connecting nerves;

- $\quad$ Systemic disorder, a problem of the body other than the head and brain;

- Vascular disorder, or blood flow problems.

A majority of balance disorders involve the vestibular system in the inner ear, hence are also called vestibular (or inner ear) disorders. Some common symptoms of a vestibular disorder are: vertigo, imbalance, jumpy vision, motion sickness. These symptoms may be quite mild, lasting minutes, or quite severe, resulting in total disability. Because the vestibular system interacts with many other parts of the nervous system, symptoms may also be experienced as problems with vision, muscles, and thinking, and memory [4]. In many cases, the cause lies in the sensory hair cell death in the vestibular organs and vestibular sensation is irreversibly lost due to: viral infections, tumors, trauma to the vestibular organs due to a head injury, toxic exposure to medication/treatment and aging.

According to studies from the National Institute of Health [5], over 90 million Americans will suffer and seek attention for balance disorders at least once in their lifetime. At least 2 million Americans suffer chronic impairment due to balance disorders, resulting in annual costs exceeding 1 billion dollars US.

It may be possible one day to regenerate hair cells and encourage self-repair, but this remains a distant goal at present [6]. Currently, the most promising hope for hair cell death seems to be a vestibular prosthesis, i.e., vestibular equivalent of a cochlear implant. A vestibular prosthesis, as defined in our research, is proposed to restore some of the vestibular sensation lost as a result of the hair cell dysfunction, based on MEMS and micro-electrode technologies.

\section{The Inner Ear}

The inner ear is a combination of the auditory and vestibular systems. The function of the auditory system is to detect sound, while the function of the vestibular system is to detect 6 DOF (degrees-offreedom) spatial information: 3 DOF linear acceleration and 3 DOF angular acceleration. The rotational movement of our body, such as when we turn our head, is detected via sensing of angular acceleration, whereas the translational movement is detected via sensing of linear acceleration.

The inner ear consists of bony (osseous) labyrinth located within the temporal bone, and a membranous labyrinth suspended within the osseous labyrinth and made up of continuous interconnected sacs and tubes. Between the osseous labyrinth and the membranous labyrinth is the perilymph, a fluid secreted by the cells lining the osseous canals. The membranous labyrinth is filled with the endolymph, a second fluid that together with the perilymph provides a medium through which we are able to process acoustic and balance information.

The osseous labyrinth can be divided into three distinct structures, each of which has a particular function: the vestibule, cochlear and semicircular canals. The cochlear is part of the auditory system, whereas the vestibular system consists of the vestibule and semicircular canals. The location of these structures within the inner year is represented in Figure 1.

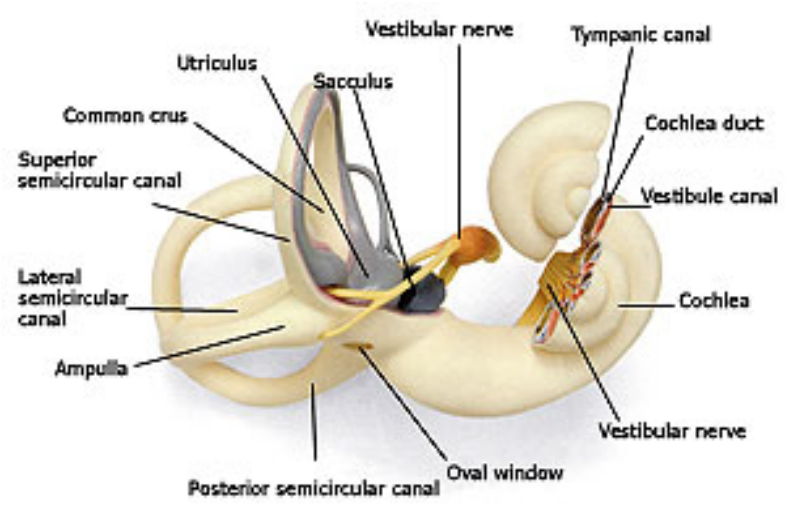

Figure 1. Inner ear

\subsection{Auditory System and Cochlear Implants}

A cochlear implant is an electronic device designed to help severely to profoundly deaf individuals who gain little or no benefit from hearing aids. Profound deafness is most often characterized by a lack of hair cells, which are the transducing medium between the pressure waves of sound, and electrical impulses, which are the neural signals sent to the brain. If the hair cells are damaged or destroyed, the ability to create neural signals is severely reduced or eliminated all together.

As shown in Figure 2, a cochlear implant consists of two main parts: an internal implanted part called the implant, and an external part known as the speech processor. Sound is picked up by a directional microphone and turned into an electrical signal. This signal goes to the speech processor where it is "coded" (digitized into a special pattern of electrical pulses). These coded pulses are sent to the transmitter and are then sent across the intact skin (by radio frequency waves) to the internal implant. The implant sends a 
pattern of electrical pulses to the micro-electrodes, which is inserted into the cochlea. The auditory nerve picks up these tiny electrical pulses and sends them to the brain.

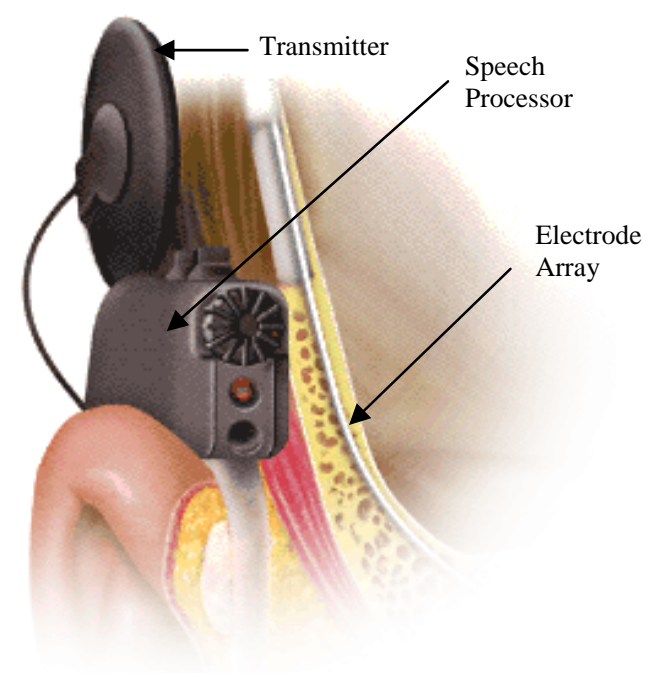

Figure 2. MED-EL's cochlear implant (from [7])

The function of a cochlear implant is then to stimulate the auditory nerve with the electrical equivalent of healthy auditory stimulus in such a way that the brain correctly interprets the signals as sound, thus mimicking the normal function of the auditory system. The implant bypasses the ear's malfunctioning mechanisms (hair cells) and provides an equivalent electrical replacement. Auditory nerve fibers in the cochlear are stimulated by the micro-electrode array. The cochlear implants are getting better every day as micro-electrodes become smaller, sound-processing electronics and surgical implantation procedures are simplified.

The micro-electrode array for cochlear implants has the most common form of a small spiral, along which are an exposed series of pads (electrodes) that ring the circumference of the spiral's length (see Figure 3). The number of electrodes corresponds to the number of channels of the array, and therefore is directly related to the number of frequencies that can be mimicked by the array. Each electrode excites a discrete batch of auditory nerve fibers along the length of the cochlea.

The number of channels in an electrode array has been known to vary from 4 to 24. Each channel transmits an electrical pulse from the receiverstimulator. A healthy individual can translate acoustic energy into electrical signals carried to the brain by the 30,000 fibers of the auditory nerve. Within the cochlea, the basilar membrane receives the acoustic energy via vibratory translation through the outer and middle ear. The vibrations take the form of a traveling wave along the membrane.

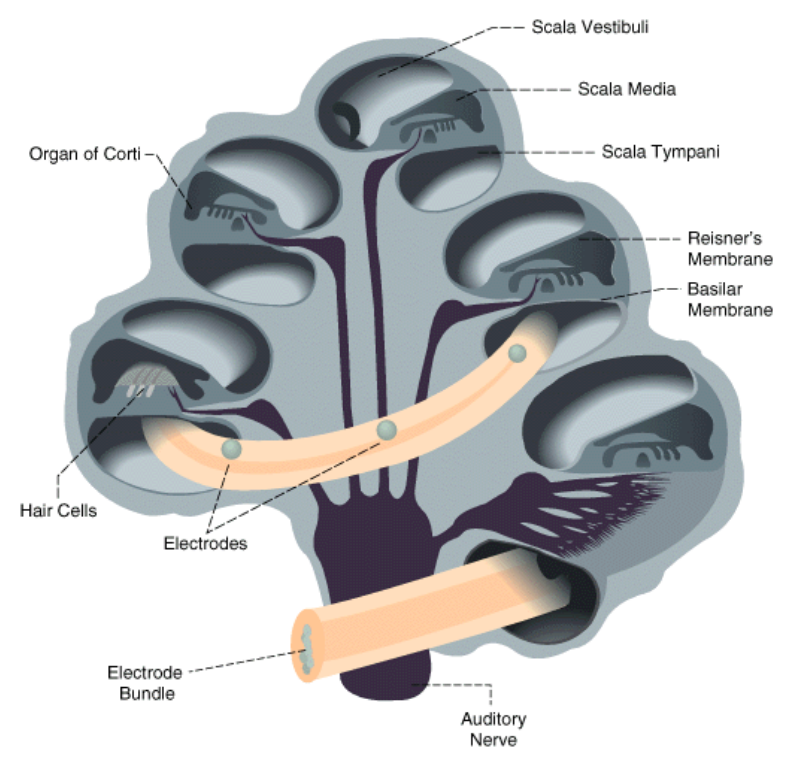

Figure 3. Electrodes within cochlea (from [8])

The energy of the traveling wave varies according to frequency. Low frequencies result in a maximum membrane displacement near the apex of the cochlea. High frequencies have a maximum displacement near the base. Varied frequencies result in maximum displacements of the basilar membrane along the corresponding areas of the cochlea.

The micro-electrode array excites the nerve fibers directly without the transducing medium of the hair cells. The fluid of the medium in which the array is inserted, the perilymph, serves as the conduction pathway between the electrode and the auditory nerve fibers.

Now, our interests in using implantable microelectrodes for vestibular prosthesis naturally arises from the advancement and success in the cochlear implant technology presented in this section. Obviously, a close attention to this technology is critical for the design of a viable vestibular implant. The subsequent section presents a brief description of the anatomy and physiology of the vestibular organs relevant to vestibular prosthetics.

\subsection{Vestibular System}

The vestibule and semicircular canals of the vestibular system are shown in Figure 1. Within the vestibule are two membranous sacs called the saccule and utricle, vestibular organs that are sensitive to gravity and translational movement. Together, the utricle and saccule detect linear accelerations. The utricle lies 
horizontally in the ear and detects motion in the horizontal plane. The saccule lies vertically and it detects motion in the sagittal plane (up and down, forward and back). The integrated role of these two organs is to keep the body oriented with respect to gravity. The utricle and saccule each have a sheet of hair cells called the macula. Within each macula the cilia of the hair cells are embedded in a gel-like substance and covered with small sheets of calcium carbonate crystals called otoliths (see Figure 4). The crystals have a higher density than the surrounding tissues and are sensitive to displacement. When we move our head from side to side, the inertia of the otolith gel mass drags on the hair gels. During constant motion the otoliths reach equilibrium and we no longer perceive the motion.

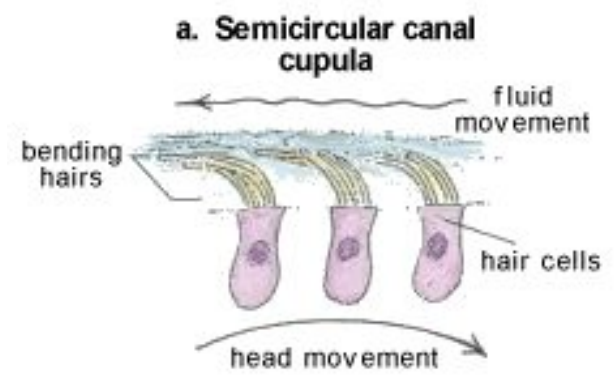

b. Utricle and Saccule

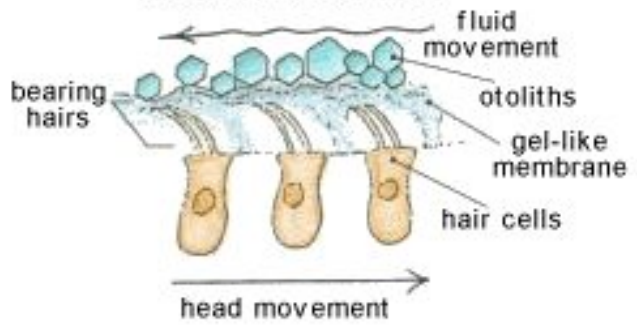

Figure 4. Vestibular organs (from [9])

The vestibular system detects angular acceleration within the semicircular canals. In addition to the utricle and saccule, each inner ear has a set of three semicircular canals, the superior, lateral and posterior semicircular. As shown in Figure 1, the three are roughly perpendicular and each corresponds to one of the three dimensions in space. Within each semicircular canal loop is an ampulla containing receptors that detect accelerations in the plane of the canal (see Figure 5).

The ampulla is a small swelling containing hair cells, arranged in a single tuft that projects into a gelatinous mass called the cupula. Inertia of the fluid in the canal moves the cupula when the head rotates (in the respective plane of the canal). The canals on either side of the head operate in a push-pull rhythm; during movement in a plane one side of the head will register a pull on the cupula and the other a push.

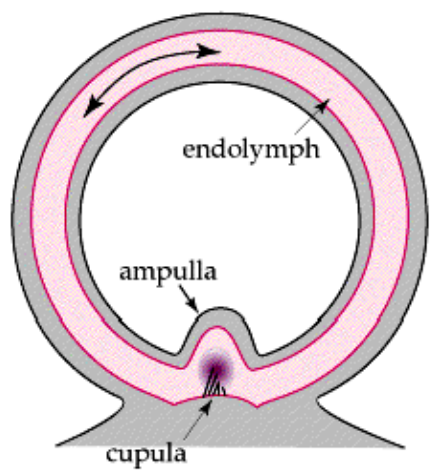

Figure 5. Semicircular canal (from [10])

The otolith organs and the semicircular canals have receptor cells equipped with an auxiliary structure within which the stereocilia of the hair cells is in contact. When the auxiliary structure moves due to inertial forces the hairs are displaced and the hair cell is activated.

Each vestibular hair cell has about 10 100 stereocilia and one kinocilium that form a bundle. The stereocilia are small hairs interconnected by small fibers called subapical bands that extend down the length of the cilia. The stereocilia vary in length ranging from smallest to highest across the bundle and tend to lie in a hexagonal array, ending at the kinocilium, which is usually aligned with the tallest stereocilia. Each stereocilia is connected to the next tallest neighbor by a top link.

One of the considerations with a vestibular implant regarding stimulation is whether an electrode is required for individual organs of the vestibular system. Both the utricle and saccule have different positions in space. It is likely that a multi-conductor electrode similar to those used in cochlear implants could be inserted into the utricle and saccule for stimulation. Each semicircular canal may also require an individual electrode configuration. The number of insertions would also depend on the extent of the dysfunction; for example, it may not be necessary to insert electrodes into all three of the semicircular canals.

\section{Vestibular Prosthesis}

The entire vestibular system in the inner ear is about the size of a marble. Ideally, the vestibular prosthesis will be of a similar size depending on where it would be placed, i.e., as small as possible if the residency of the implant (or part of it) is within the skull itself. 
According to a recent review article [6] on current state of vestibular technology, there seems to be two potential stimulation mechanisms that can be interfaced with a vestibular motion sensor: (i) neural stimulation using micro-electrodes and (ii) micromechanical stimulation using MEMS devices. While our focus falls into the first category, direct micromechanical stimulation can also be used to excite individual vestibular organs with vestibular hyperfunction - a condition in which a subset of haircells remains functional but the organs are deficient in overall gain. In principle, the implanted micromechanical stimulator (i.e., a micro beam) would provide a mechanically amplified stimulus by directly pressing on the hair-cells.

If micro-electrodes are used, due to the fact that the varied organs comprise the vestibular system, it is most likely that more than one electrode array is required, one geometry for the semicircular canals, and at least one other geometry for the otolith organs (the utricle and saccule). In comparison, cochlear electrode arrays represent a much simpler problem in terms of geometry due to the linear nature of the cochlea, i.e., a single electrode array with the multiple micro-electrodes arranged in series is capable of stimulating the auditory nerve to represent sounds. However, the number of required micro-electrodes in a vestibular electrode array would be greatly reduced compared to that of a cochlear electrode array. This is because the cochlear electrode array requires many channels to cover the wide frequency range of audible sound, whereas the number of channels required by the vestibular electrode array implanted in each vestibular organ would correspond to the number of DOF motion information provided by that organ. The different functional nature of the arrays required for stimulating the vestibular system result in the following challenges:

- Providing full stimulation to the vestibular system

- Having an adequate number of electrodes to stimulate the utricle, saccule and semicircular canals (1 to 5 arrays)

- Creating separate electrode arrays capable of stimulating the utricle, saccule and semicircular canals.

- Having a connection capability to interconnect the electrodes to the conducting cables that transmit electrical pulses.

The partnership between UVic and Epic Biosonics presents an opportunity to address the challenges listed above, making the possibility of an implantable vestibular prosthesis a reality.

\subsection{Current Technology}

Literature survey shows that there are a number of ongoing projects that suggest potential solutions to balance disorders. Many of these projects involve amplification of the proprioception system, for example, inserts that go into footwear that sends out a vibration to the foot when balance is threatened, vests utilizing vibrotactile pads that respond to "body-tilt" measurements (e.g. [11]). These devices address rehabilitation of the vestibular system, not a (permanent) replacement, and are intended to reduce postural sway while subjects are standing still.

A research group at UC Irvine has recently developed an integrated 3-axes MEMS gyroscope sensing circuitry that can mimic the function of semicircular canals [12]. They are currently working towards creating a complete MEMS-based vestibular implant. We envision our vestibular implant to consist of two parts: an external MEMS-based signal processor for 6 DOF motion sensing and internal implanted part containing the vestibular electrode array circuitry.

\section{Electrode Array Circuitry Basics}

All implantable electrode array systems have three major components, the leads, the power supply and the connecting cable between the two. The power supply is a critical component of any implant system, providing the electrical pulses to stimulate the target tissue or nerve. Generally there are two types of power supply systems, implantable and Radio Frequency (RF). The implantable power supply is a self-sufficient system, able to provide low power neurostimulation without external power requirements.

The second type of power supply, used for highpower implant requirements, is the RF system. Usually these systems are composed of an implantable (receiver) and external (transmitter) system. The signal is transmitted through the skin from the transmitter to the receiver which conducts the signal through the conducting cable to the lead.

The lead delivers the stimulation pulses to the target tissues, a conductor with exposed electrodes that deliver the pulses. The geometry of the lead is entirely dependent on the number of conductors the cable is capable of containing without sacrificing flexibility and biocompatibility. Many leads on the market today are manufactured for spinal cord or peripheral nerve stimulation and are constrained by the number of conductors the lead can carry. The more conductors within the cable, the more electrodes on the lead, as each conductor represents a discrete electrode.

The cable connects the power supply to the lead. The number of discrete conductors within the cable is often limited by the need for flexibility and size constraints. A critical area of importance in 
implantable systems is the ability to connect the cable to the lead and power supply in a biocompatible robust manner.

The limitations around conventional circuitry center on issues such as an inability to manufacture leads with a large number of electrodes, high flexibility, low weight and smooth geometry to minimize tissue reactions to the device.

\subsection{Epic's Circuitry}

Epic Biosonics has designed a new generation of flexible platinum circuitry capable of generating electrodes that represent an extremely functional and economical alternative to the components on the market today. Epic's primary goal is to become a leading provider of implantable electrode arrays and microcables for the neurotechnology industry. Epic has exclusively licensed this technology in the field of hearing implants.

The technology platform Epic has developed represents the ability to design and develop implantable electrode arrays over a range of applications which include (and are not limited to):

- Neurostimulation

- Neuromodulation for chronic pain management

- Functional electrical stimulation (FES)

The technology revolves around the ability to encapsulate micro-structured platinum circuitry in a fluropolymer film. This unique processing method has resulted in highly structured and flexible encapsulated circuits. This 2D film can also be manipulated into a $3 \mathrm{D}$ structure to meet device functional requirements.

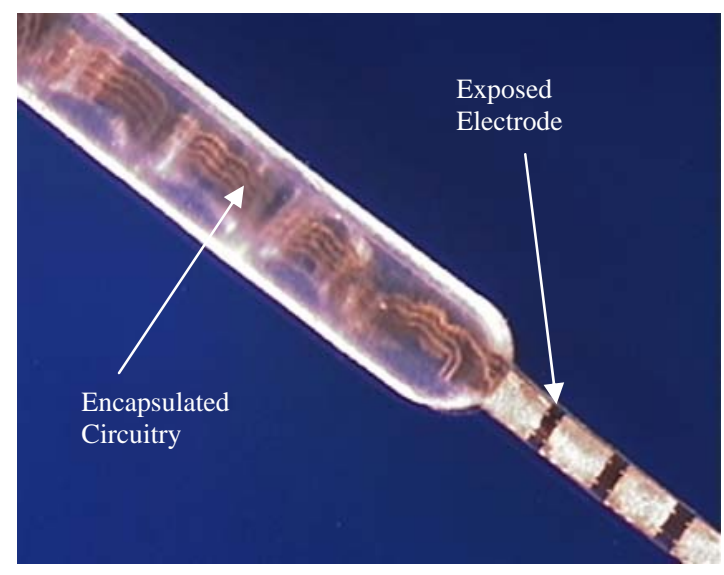

Figure 6. Encapsulated Epic circuit

The significance of this circuitry is the ability to create highly flexible micro-conductors, encapsulated and formed to meet device requirements. This technology is currently being applied to cochlear implants, in particular the stimulating electrode array, representing the possibility of the most technologically advanced micro-arrays available. Some of the capabilities of Epic's circuitry include:

- Small in size: The platinum conductors are typically 30 to 70 microns wide, with 20-30 micron spacing. The conductors are fabricated in such a way that the conversion from a 2D film to a $3 \mathrm{D}$ finished array only increases the robustness of the product.

- Design flexibility: The number of electrodes is more likely to be hindered by the size of the target tissue than the fabrication process.

- Mechanical flexibility: The micro-cables are designed to be able to withstand 360 degree twists, elongation over $115 \%$ and lateral and horizontal flexure through millions of cycles.

- Longevity: On-going accelerated aging tests in saline solution indicate the components are well on their way to meeting Epic's 20 year insulation survival criterion.

- Biocompatibility: All materials have been chosen for their biocompatible properties (platinum, fluropolymer, silicone).

- Batch fabrication: Fabrication of the devices using Epic's patented process enables cost efficient and repeatable electrode fabrication [13].

- Rapid prototyping: The ability to go from concept to prototype is limited only by the time it takes to create a CAD file and the time to make a phototool.

The creation of a set of electrode arrays for stimulation of the vestibular system is only limited by the design geometry. The technology also allows the possibility of numerous electrodes, limited only by the surface area of the target tissues and the desired properties of the cable connection. Most importantly however, the technology is available to fabricate micro-electrode systems with variable geometry, high numbers of electrodes and with high reproducibility.

Epic's abilities also bridge the connection of cables to the arrays. This interconnection between array and cable must meet high biocompatibility and robustness criteria. Epic is in the process of developing a method with which to attach various cable systems to their electrode arrays, a highly delicate and critical issue for all implantable electrodes. The development of a solid interconnection strategy would represent a significant milestone, further strengthening and expanding the possibilities and applications of implantable circuitry.

The delicate nature of the device represents a challenge for interconnection strategies. Attaching the platinum conductors to the cable systems without melting or otherwise effecting the polymer insulation is a significant challenge. Ideally the connection 
between the conductors and the electrodes will be made of a continuous blend between the two. Epic is currently researching methods to attach the insulated conductors to the electrodes to provide a continuous and secure electrical and mechanical connection.

\section{Future Work}

Utilizing MEMS technology and micro-electrode array circuitry and interconnection capabilities, the possibility of creating a vestibular prosthesis hinges more on the issue of how to stimulate the vestibular nerve in such a way as the device stimuli are accurately combined with visual and proprioception stimuli to resilt in coherent balance signals. Research into the number and geometry of electrodes required for proper stimulation is needed, whicu is dependant on where the stimulation would occur.

Epic is well on their way to presenting a viable micro-electrode and interconnection technology, and MEMS technology is developing rapidly to become a main innovation in the medical field, responding to the demands of increasingly smaller biomedical devices. Using MEMS technology, together with Integrated Circuit (IC) technology, micromachining can shrink such vestibular sensors by orders of magnitude, reduce the fabrication costs and allow the electronics to be integrated on the same silicon chip [14].

We are in the process of building a prototype 6 DOF motion sensing device that will mimic not only the functions of the semicircular canals, but those of the utricle and saccule as well. This prototype should provide 6 DOF high resolution, high fidelity and high bandwidth motion information accurately representing the motion of the body. The circuitry of our prototype device will include commercially available MEMS accelerometers and gyroscopes provided that will be implemented on a Printed Circuit Board (PCB), along with associated electronics. These commercialized MEMS devices are being used for demonstration purposes in this proof-of-concept stage of our research.

In order to mimic the response of the vestibular organs, the output voltages of the accelerometers and gyroscopes need to be conditioned such that they produce signals similar to these natural organs. Requirements for the motion sensor performance can be estimated from the vestibular organ performance data already available in the literature (e.g. [15]). We are currently developing an accurate simulation model to investigate these issues, which will allow us to design proper signal conditioning electronics. Our long-term goal is to build a totally implantable vestibular prosthesis with a 6 DOF motion sensor ona-chip, connected to a set of vestibular electrode arrays for the direct nerve stimulation.

\section{References}

[1] J. Cavuoto, "Neural Engineering's Image Problem", IEEE Spectrum, pp. 32-37, April 2004.

[2] Hendrick Balance Center, Balance Disorders [online document] available from WWW [cited November 12, 2003]:

$<$ http://www.hendrickhealth.org/balance.disorders.htm $>$.

[3] National Institute of Health, Balance Disorders, NIH Publication No. 00-4374, January 2000.

[4] Vestibular Disorders Association, Vestibular Disorders: An Overview [online document], available from WWW [cited May 29, 2004]:

<http://www.vestibular.org/overview.html>

[5] National Institute of Health, National Strategic Research Plan: Language and Language Impairments, Balance and Balance Disorders, Voice and Voice Disorders.

[6] D.M. Merfeld and R.D. Rabbitt, "Vestibular Prosthetics”, Neuroprosthetics, K. Horch et al. (ed.), World Scientific Pub. Co., 2003.

[7] MED-EL Inc., How a Cochlear Implant Works [online document], available from WWW [cited May 29, 2004]:

$<$ http://www.medel.com/ENG/INT/10_Understanding_ CI/20_How_a_CI_works/090_how_ci_works.asp\#>

[8] D.K. Eddington and M.L. Pierschalla, "Cochlear Implants: Restoring Hearing for the Deaf", The Harvard Mahoney Neuroscience Institute Letter on the Brain, 3(4), fall 1994.

[9] L.R. Young, "Human Sensory and Balance System" [online document], Human Physiology in Space, B.F. Lujan and R.J. White (eds.), available from WWW [cited May 29, 2004]:

<http://www.nsbri.org/HumanPhysSpace/focus7/ep_str ucture.html>

[10] S. Essig and N. Negoi, The Human Vestibular System [online document], fall 1998, available from WWW [cited May 29, 2004]:

<http://paperairplane.mit.edu/16.423J/Space/SBE/neuro vestibular/NeuroVestibular/2_Physiology/PhysSub3.ht $\mathrm{ml}>$

[11] C. Wall and M.S. Weinberg, "Balance Prostheses for Postural Control”, IEEE Engineering in Medicine and Biology Magazine, pp. 84-90, March/April 2003.

[12] J. Liu and A.M. Shkel, "An Electronic Prosthesis Mimicking the Dynamic Vestibular Function", 2003 IEEE Sensors Conference, pp. 996-1001, Toronto, Canada, September 2003.

[13] P.G. Berrang, H.V. Bluger, H. Klosowski and A.J. Lupin, Modiolar Hugging Electrode Array, U.S. Patent 6,374,143.

[14] A.M. Shkel, "Micromachined Gyroscopes: Challenges, Design Solutions, and Opportunities", 2001 SPIE Annual International Symposium on Smart Structures and Materials, Newport Beach, CA, March 2001.

[15] C. Wall et al., "Balance Prosthesis Based on Micromechanical Sensors Using Vibrotactile Feedback of Tilt”, IEEE Trans. on Biomedical Engineering, 48(10), pp. 1153-1161, October 2001. 